\section{Das Bronchialkarzinom des jungen Erwachsenen}

Zusammenfassung: Das Bronchialkarzinom des jungen Erwachsenen wurde in seinem Erscheinungsbild hinsichtlich relativer Inzidenz, Verteilung von Stadien und Morphologie und Prognose unterschiedlich geschildert in Publikationen aus verschiedenen Regionen der Welt. Wir haben retrospektiv die Daten der Tumordokumentation der Lungenklinik Heckeshorn in Berlin von 1986 bis 1995 zu dieser Fragestellung ausgewertet. 106/4939 Patienten (2,1\%) waren 40 Jahre alt oder jünger. Der Frauenanteil war mit 42 vs. $29 \%$ in der jüngeren Gruppe deutlich höher. Das Adenokarzinom war der führende Subtyp in der jüngeren Gruppe (33 vs. 24\%). Bei den nichtkleinzelligen Karzinomen zeigten die jüngeren Patienten eine Verschiebung zu den inoperablen Stadien IIIB und IV (71 vs. $46 \%$ ). In den Stadien I bis IIIA wurden die Patienten bis $\mathbf{4 0}$ Jahre mit nichtkleinzelligen Tumoren wesentlich häufiger operiert (90 vs. $49 \%$ ), jedoch ergab sich für die Gesamtgruppe mit dieser Morphologie kein Überlebensvorteil für die jüngeren Patienten (302 vs. 314 Tage). Zusammenfassend zeigte sich bei durchaus unterschiedlichem altersbezogenen Erscheinungsbild des Bronchialkarzinoms in unserem Patientenkollektiv keine bessere Prognose für jüngere Patienten.

Lung Cancer in Young Adults: Lung cancer in young adults has been differently described in publications from various regions of the world, in respect of relative incidence, distribution of morphology and stages, and prognosis of the disease. We analysed retrospectively the data from the cancer registry of the Lungenklinik Heckeshorn in Berlin between 1986 and 1995 with regard to this topic. $106 / 4939$ patients were 40 years old or younger $(2.1 \%)$. The proportion of female patients was higher in the younger group (42 vs. $29 \%$ ). Adenocarcinoma was the leading subtype in young patients (33 vs. $24 \%$ ). The younger group showed a higher proportion of inoperable stages IIIB and IV for non-small cell lung cancer (71 vs. $46 \%$ ). Younger patients with operable stages I to IIIA non-small cell lung cancer underwent surgery much more often than patients older than 40 years ( 90 vs. $49 \%$ ), but for all patients with this morphology there was no difference in survival between younger and older patients (302 vs. 314 patients). We conclude that lung cancer presents a clearly different clinical picture in younger patients but not a better prognosis.

Pneumologie 53 (1999) 480-484

(c) Georg Thieme Verlag Stuttgart · New York
N. Schönfeld, T. Lienert, M. Serke, D. Kaiser ${ }^{1}$,

R. Loddenkemper

Pneumologische Abt. II und

${ }^{1}$ Thoraxchirurgische Abteilung der Lungenklinik Heckeshorn, Berlin

\section{Einleitung}

Während das Bronchialkarzinom in der Vergangenheit größtenteils eine Erkrankung der männlichen Bevölkerung im höheren Lebensalter darstellte, hat der Anteil der weiblichen Patienten in jüngster Zeit deutlich zugenommen [8]. Daneben gibt es eine weitere Gruppe, in der sich die veränderten Inzidenzdaten des Bronchialkarzinoms widerspiegeln: Patienten in der dritten oder vierten Lebensdekade. In verschiedenen retrospektiven Studien wurde das klinische Erscheinungsbild des Bronchialkarzinoms beim jungen Erwachsenen hinsichtlich der Inzidenz, der Verteilung von histologischen Subtypen und Stadien sowie der Prognose untersucht [1$3,6,9,11-13]$. Hierbei zeigten sich zum Teil erhebliche Unterschiede für alle genannten Kenngrößen, die am ehesten auf regionale, d.h. populationsabhängige Faktoren zurückzuführen sind. Aus Mitteleuropa bzw. Deutschland wurden vergleichbare Daten dazu noch nicht publiziert, unabhängig von den hierzulande laufend berichteten epidemiologischen Daten des Statistischen Bundesamtes, der Landesämter und im besonderen des Saarländischen Krebsregisters sowie den Zahlen aus der ehemaligen DDR [10].

\section{Patienten und Methoden}

Die Lungenklinik Heckeshorn, gelegen auf dem Gebiet des ehemaligen Westteils von Berlin, ist eine onkologische Schwerpunktklinik im Verbund des Tumorzentrums Berlin e.V. Seit Beginn des Jahres 1986 liegt eine vollständige Erfassung der in der Klinik behandelten Patienten mit Tumorerkrankungen vor. Im Zeitraum von 1986 bis 1995 wurden 4939 Patienten mit definitiver histologischer oder zytologischer Diagnose eines Bronchialkarzinoms registriert, deren Daten wir retrospektiv ausgewertet haben. Unterschiede in der generellen Gruppenverteilung wurden mittels Chi-Quadrat-Test berechnet; altersbezogene Unterschiede zwischen einzelnen Gruppen wurden nur angegeben, wenn dies aufgrund der Gruppengröße auch sinnvoll zu berechnen war. Die medianen Überlebenszeiten wurden nach der Kaplan-MeierMethode ermittelt und mit dem Logranktest verglichen. Alle angegebenen p-Werte sind zweiseitig.

\section{Ergebnisse}

Die Gesamtgruppe umfaßte 1454 Frauen und 3485 Männer mit einem Altersmedian von 64,2 Jahren (Altersspanne 14,1 bis 92,4 Jahre). 106 der 4939 Patienten (2,1\%) waren 40 Jahre oder jünger. Der Frauenanteil war mit $42 \%$ in dieser Gruppe signifikant höher als bei den Patienten über 40 Jahre $(29 \%$, 
$p=0,006)$. Bei der prozentualen Verteilung der histologischen Subtypen zeigte sich ein deutlich höherer Anteil der Adenokarzinome in der Gruppe junger Patienten (33 versus $24 \%)$, was in erster Linie auf den hohen Anteil der Adenokarzinome bei jungen weiblichen Patienten zurückzuführen war, wenngleich das Adenokarzinom auch bei Männern bis 40 Jahren der häufigste Tumorsubtyp war (Tab. 1, Abb.1 -3).

Ein komplementäres Bild hierzu ergab sich bei den Plattenepithelkarzinomen, die in der jüngeren Patientengruppe mit $10 \%$ vertreten waren, während der Anteil im höheren Lebensalter bei 34\% unter Männern und bei 18\% unter Frauen lag. Erwartungsgemäß war der Anteil der Karzinoide in der jüngeren Population erheblich höher als in späterem Alter (16 versus $1,2 \%)$. Niedriger lag dagegen der Anteil der kleinzelligen Bronchialkarzinome im jüngeren Alter (9 versus 19\%).

Hinsichtlich der Stadienverteilung ergab die Unterscheidung zwischen jüngeren und älteren Patienten für die histologisch heterogene Gesamtgruppe ein Überwiegen der Stadien I bis IIla (39 versus 43\%) im höherem Alter (Tab. 2). Aufschlußreicher erwies sich hier die Analyse der nichtkleinzelligen

\begin{tabular}{|c|c|c|c|c|c|c|c|c|}
\hline \multirow[t]{2}{*}{ Histologie } & \multicolumn{2}{|c|}{$\begin{array}{l}\text { Frauen } \\
\text { bis } 40 \text { Jahre }\end{array}$} & \multicolumn{2}{|c|}{$\begin{array}{l}\text { Männer } \\
\text { bis } 40 \text { Jahre }\end{array}$} & \multicolumn{2}{|c|}{$\begin{array}{l}\text { Frauen } \\
\text { über } 40 \text { Jahre }\end{array}$} & \multicolumn{2}{|c|}{$\begin{array}{l}\text { Männer } \\
\text { über } 40 \text { Jahre }\end{array}$} \\
\hline & $\mathrm{n}$ & & $\mathrm{n}$ & & & & & \\
\hline adenoid & 18 & 40,9 & 17 & 27,4 & 405 & 28,7 & 753 & 22,0 \\
\hline bronchiolo-alveolär & 3 & 6,8 & 1 & 1,6 & 53 & 3,8 & 54 & 1,6 \\
\hline plattenepithelial & 4 & 9,1 & 7 & $11,3^{*}$ & 248 & 17,6 & 1151 & 33,6 \\
\hline kleinzellig & 3 & $6,8^{*}$ & 6 & 9,7 & 321 & 22,8 & 636 & 18,6 \\
\hline mittelgroß/großzellig & 6 & 13,6 & 15 & 24,2 & 204 & 14,5 & 511 & 14,9 \\
\hline Karzinoide & 9 & $20,5^{*}$ & 8 & $12,9^{*}$ & 35 & 2,5 & 24 & 0,7 \\
\hline sonstige & 1 & 2,3 & 8 & 12,9 & 144 & 10,2 & 294 & 8,6 \\
\hline gesamt & 44 & 100 & 62 & 100 & 1410 & 100 & 3423 & 100 \\
\hline
\end{tabular}

Tab. 1 Histologische Verteilung aller malignen Lungentumoren in der Lungenklinik Heckeshorn 1986 - 1995 nach Geschlecht und Alter $(n=4939)$; $p<0,001$ bezüglich der Unterschiede in der Gesamtverteilung, ${ }^{*} \mathrm{p}<0,05$ für Unterschiede innerhalb einer morphologischen Gruppe.

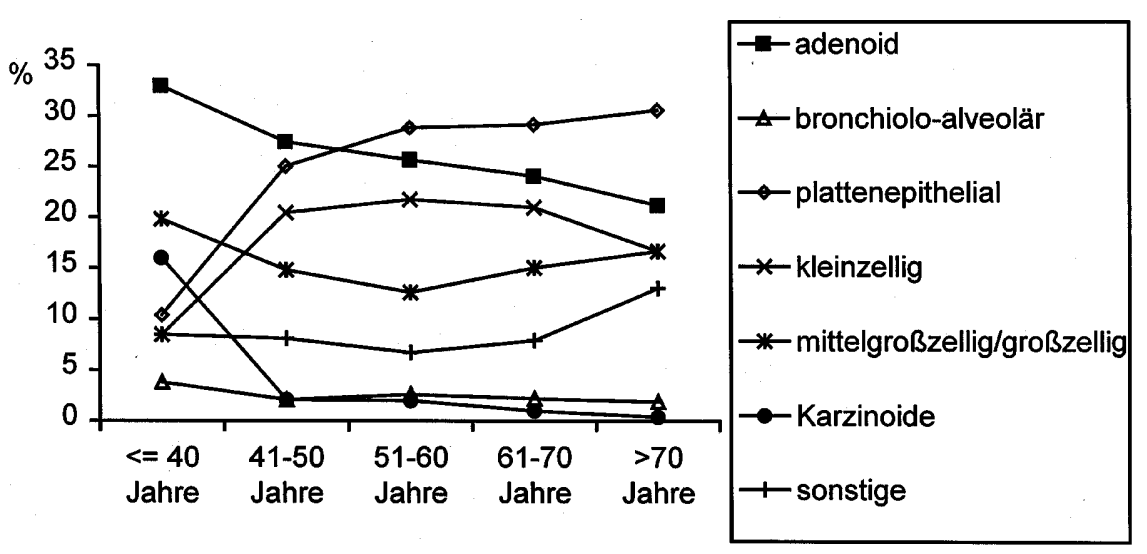

Abb. 1 Histologische Verteilung aller malignen Lungentumoren nach Altersgruppen, Lungenklinik Heckeshorn $1986-1995$ ( $n=4939) ; p<0,001$ bezüglich der Unterschiede in der Gesamtverteilung.

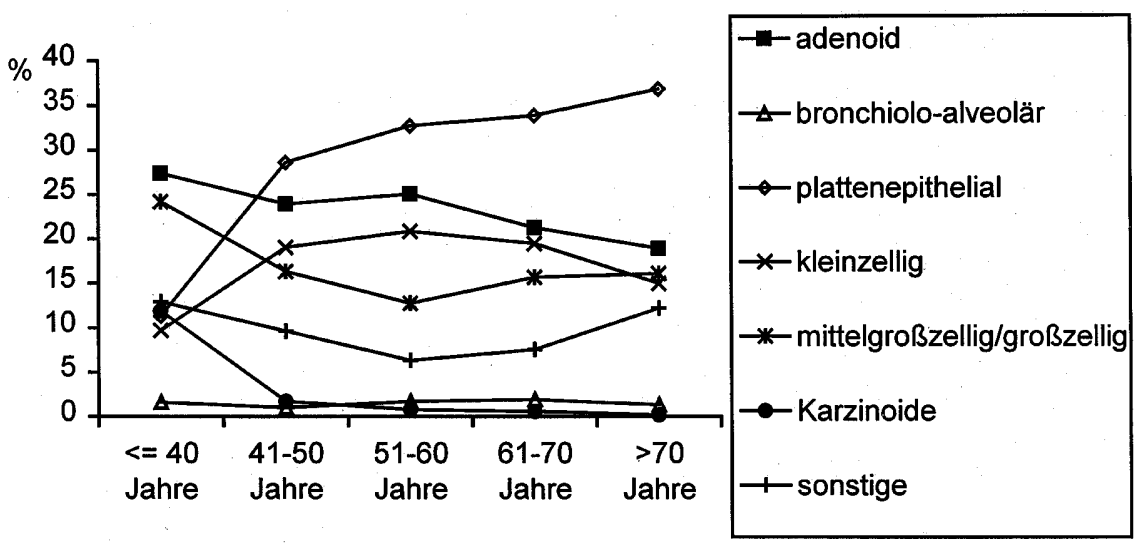

Abb. 2 Histologische Verteilung aller malignen Lungentumoren in der Lungenklinik Heckeshorn 1986-1995 nach Altersgruppen bei Männern ( $n=3485)$; $p<0,001$ bezüglich der Unterschiede in der Gesamtverteilung. 


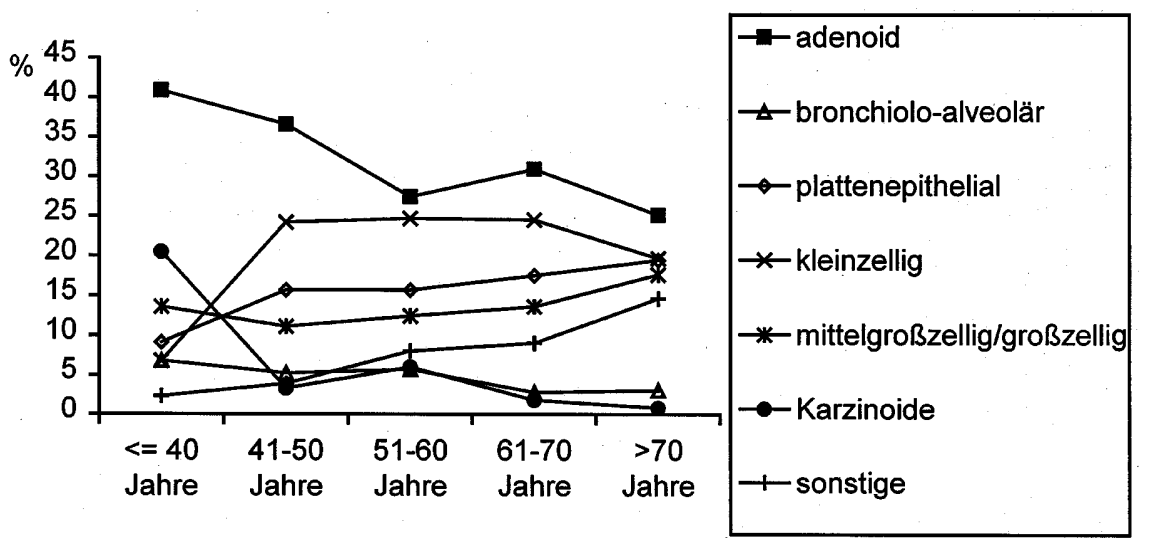

Abb. 3 Histologische Verteilung aller malignen Lungentumoren nach Altersgruppen bei Frauen ( $n=1454$ ), Lungenklinik Heckeshorn 1986-1995; $p<0,001$ bezüglich der Unterschiede in der Gesamtverteilung.
Karzinome als der größten Untergruppe, die eine noch deutlichere Verschiebung zugunsten der fortgeschrittenen und praktisch immer inoperablen Stadien IIIb und IV in der jüngeren Patientengruppe ergab (71 versus $46 \%, \mathrm{p}<0,001$ ) (Tab. 3).

Bei der Analyse der jemals im Krankheitsverlauf durchgeführten Therapien für alle Patienten zeigte sich, daß in den Stadien I bis IIla junge Patienten signifikant häufiger operiert (90 versus $47 \%, p<0,001)$, chemotherapiert (29 versus $15 \%$, $\mathrm{p}=0,014$ ), aber dafür seltener bestrahlt wurden (42 versus $68 \%, \mathrm{p}<0,001)$. Die Ergebnisse für die Patienten mit nichtkleinzelligen Karzinomen sind gesondert in Tab. 4 aufgeführt. In den Stadien IIIb und IV zeigten sich dagegen schwächere Unterschiede (Tab. 5).

Tab. 2 Stadienverteilung aller Bronchialkarzinome in der Lungenklinik Heckeshorn 1986-1995 $(n=4939) ; p=0,477$ bezüglich der Unterschiede in der Gesamtverteilung.

\begin{tabular}{llllllll}
\hline Stadium & \multicolumn{2}{l}{ Pat. bis 40 J. } & \multicolumn{2}{l}{ Pat. über 40 J. } & \multicolumn{2}{l}{ gesamt } \\
& n & $\%$ & n & $\%$ & n & $\%$ \\
\hline I-IIla & 41 & 38,7 & 2062 & 42,7 & 2103 & 42,6 \\
IIIb-IV & 58 & 54,7 & 2366 & 49,0 & 2424 & 49,1 \\
keine Angabe & 7 & 6,6 & 405 & 8,4 & 412 & 8,3 \\
\hline gesamt & 106 & 100 & 4833 & 100 & 4939 & 100 \\
\hline
\end{tabular}

Tab. 3 Stadienverteilung nichtkleinzelliger Bronchialkarzinome, Lungenklinik Heckeshorn 1986- 1995 ( $n=3451)$; $p<0,001$ bezüglich der unterschiedlichen Stadienverteilung zwischen den Altersgruppen.

\begin{tabular}{llllllll}
\hline Stadium & \multicolumn{2}{l}{ Pat. bis 40 J. } & \multicolumn{2}{l}{ Pat. über 40 J. } & \multicolumn{2}{l}{ gesamt } \\
& n & $\%$ & n & $\%$ & n & $\%$ \\
\hline I-IIIa & 20 & 27,8 & 1690 & 50,0 & 1710 & 49,5 \\
IIIb-IV & 51 & 70,8 & 1557 & 46,1 & 1608 & 46,6 \\
keine Angabe & 1 & 1,4 & 132 & 3,9 & 133 & 3,9 \\
\hline gesamt & 72 & 100 & 3379 & 100 & 3451 & 100 \\
\hline
\end{tabular}

Hinsichtlich der Prognose deutete der altersbezogene Vergleich mittels Kaplan-Meier-Analyse keinen Überlebensvorteil für die jüngeren Patienten mit nichtkleinzelligen Karzinomen an (Tab.6). Die weitere Differenzierung nach Stadien in dieser Gruppe zeigte, daß allerdings ein nicht signifikanter Überlebensvorteil bei den frühen, noch operablen Tumorstadien I bis IIIa zu bestehen scheint (medianes Überleben: 868 versus 538 Tage, $\mathrm{p}=0,065$ ). In den fortgeschrittenen Stadien IIIb und IV konnte keinerlei Unterschied zwischen beiden Gruppen aufgezeigt werden.

Hinsichtlich relevanter Riskofaktoren (vor allem Rauchen) konnte aus den retrospektiven Daten keine hinreichende Aussage zum Verhältnis zwischen jüngeren und älteren Patienten getroffen werden.

Tab. 4 Durchgeführte Therapien nach Alter, Patienten mit nichtkleinzelligem Bronchialkarzinom im Stadium I bis Illa, Lungenklinik Heckeshorn 1986-1995.

\begin{tabular}{|c|c|c|c|c|c|}
\hline \multirow[t]{2}{*}{ Therapie } & \multicolumn{2}{|c|}{$\begin{array}{l}\text { Stadium I bis IIla } \\
\text { Pat. bis } 40 \mathrm{~J} .\end{array}$} & \multicolumn{2}{|c|}{ Pat. über 40 J. } & \multirow[t]{2}{*}{$P$} \\
\hline & $\mathrm{n}$ & $\%$ & & & \\
\hline keine Therapie & 0 & 0 & 109 & 6,4 & 0,634 \\
\hline gesamt OP & 18 & 90,0 & 838 & 49,6 & $<0,001$ \\
\hline gesamt Chemo & 6 & 30,0 & 114 & 6,7 & 0,002 \\
\hline gesamt Rad & 12 & 60,0 & 1173 & 69,4 & 0,464 \\
\hline
\end{tabular}

Tab. 5 Durchgeführte Therapien nach Alter, Patienten mit nichtkleinzelligem Bronchialkarzinom im Stadium IIIb und IV, Lungenklinik Heckeshorn 1986- 1995.

\begin{tabular}{|c|c|c|c|c|c|}
\hline \multirow[t]{2}{*}{ Therapie } & \multicolumn{4}{|c|}{ Stadium IIIb und IV } & \multirow{2}{*}{$\mathrm{P}$} \\
\hline & $\mathrm{n}$ & $\%$ & $\mathrm{n}$ & & \\
\hline keine Therapie & 10 & 19,6 & 184 & 11,8 & 0,121 \\
\hline gesamt OP & 11 & 21,6 & 196 & 12,6 & 0,084 \\
\hline gesamt Chemo & 33 & 64,7 & 570 & 36,6 & $<0,001$ \\
\hline gesamt Rad & 33 & 64,7 & 1182 & 75,9 & 0,07 \\
\hline
\end{tabular}


Tab. 6 Überleben (Medianwert in Tagen) nach Alter und Stadium beim nicht-kleinzelligen Bronchialkarzinom, Lungenklinik Heckeshorn $1986-1995$.

\begin{tabular}{|c|c|c|c|c|c|c|}
\hline & \multirow{2}{*}{$\begin{array}{l}\text { alle } \\
\text { Tage }\end{array}$} & \multirow[b]{2}{*}{$\mathrm{n}$} & \multicolumn{2}{|c|}{ Stadium I-IIIa } & \multicolumn{2}{|c|}{ Stadium IIIb-IV } \\
\hline & & & Tage & $\mathrm{n}$ & Tage & $\mathrm{n}$ \\
\hline Pat. bis $40 \mathrm{~J}$. & 302 & 64 & 868 & 18 & 247 & 45 \\
\hline Pat. über 40 J. & 314 & 3042 & 538 & 1495 & 195 & 1443 \\
\hline $\mathrm{p}$ & \multicolumn{2}{|c|}{0,999} & \multicolumn{2}{|c|}{0,065} & \multicolumn{2}{|c|}{0,411} \\
\hline
\end{tabular}

\section{Diskussion}

Beim jungen Erwachsenen ist das Bronchialkarzinom eine seltene Erkrankung. In den bisher berichteten Serien, bei denen es sich ausschließlich um retrospektive Analysen handelte, lag der Anteil der Patienten unter 40 Jahre zwischen einem und drei Prozent vom Gesamtkrankengut, in unserer Auswertung bei 2,1\% [1,6,12]. Nur in einer der Studien zu den jüngeren Patienten [9] zeigte sich das Verhältnis von Männern und Frauen bei den jungen Patienten so ausgeglichen $(1,2: 1)$ wie in unserem Kollektiv. Andere Studien ergaben zwar einen ähnlichen Trend, jedoch wurde auch über ein völliges Fehlen einer solchen altersbezogenen Verschiebung berichtet (Italien, Israel [2]). Insofern sind also regionale Verschiedenheiten zumindest bei der Interpretation jeglicher altersbezogener Daten zum Bronchialkarzinom zu berücksichtigen. Die Unterschiede ergeben sich offenbar nur zum Teil aus einem unterschiedlichen Rauchverhalten, da aus allen genannten Studien ein ähnlich hoher Anteil an Rauchern unter jüngeren wie unter älteren Patienten hervorgeht. Als weitere Einflußmöglichkeiten kommen so weite Bereiche wie Genetik, andere ökologische Faktoren oder Selektionskriterien infrage.

Ein deutlich größerer Prozentsatz an Adenokarzinomen bei Jüngeren trat bei unseren Patienten relativ eng begrenzt in der Altersgruppe unter 40 Jahren auf. Schon in der fünften Dekade blieb in unserer Statistik der dann niedrigere Anteil an der Gesamtzahl der Patienten relativ konstant, wobei wie auch in anderen Untersuchungen Frauen generell eine höhere Rate an Adenokarzinomen aufwiesen; gleichwohl können auch hier regional erhebliche Unterschiede bestehen [4]. Bemerkenswert für die Gruppe junger Patienten sowohl in unserem Kollektiv als auch anderen erscheint, daß aber der stärkere Frauenanteil nicht allein für das höhere Aufkommen an Adenokarzinomen verantwortlich ist, sondern sowohl Männer als auch Frauen hiervon relativ stärker betroffen sind. Die seit Jahren beobachtete absolute Zunahme adenoider Bronchialkarzinome - vor allem in den USA sowie in Europa in den Niederlanden - wird allerdings überwiegend auf die wachsende Zahl weiblicher Patienten zurückgeführt $[4,5,14]$.

Hinsichtlich der Stadieneinteilung ergab sich in unserer Auswertung eine Überrepräsentation lokal fortgeschrittener Tumorstadien in der Gruppe der jüngeren Patienten. Auch dieses Phänomen konnte in etwas unterschiedlicher Ausprägung von anderen Arbeitsgruppen beobachtet werden [11], betraf dort allerdings auch die Patienten mit Fernmetastasen, was bei unseren Patienten wiederum weniger deutlich erkennbar war. Es kann nur spekuliert werden, ob für die unterschiedliche Stadienverteilung nicht allein die andere Verteilung der Morphologie als Erklärung heranzuziehen ist, sondern ob vielmehr grundsätzlich die Tumoren bei jungen Patienten ein unterschiedliches biologisches Verhalten aufweisen. Die Verschleppungszeit vom Symptombeginn bis zur Diagnosestellung bei jüngeren Patienten wurde im Vergleich teils kürzer [3], teils länger [2] angegeben und scheint daher eher von geringerer Bedeutung für das Schicksal dieser Patienten zu sein. Alles in allem gibt es aber zu diesen Punkten kein ausreichendes Datenmaterial aus den bisherigen Studien, um zu sicheren Aussagen zu kommen.

Besonderes Interesse gehört im Zusammenhang mit der Stadienverteilung den Daten zum Überleben, die aus der Literatur als auch aus unseren Daten heraus keinen bzw. nur einen sehr geringen Überlebensvorteil für jüngere Patienten erkennen lassen [11]. Die im Vergleich zum Gesamtkollektiv kleine Gruppe der Operierten, unter denen die jüngeren Patienten allerdings einen deutlichen Überlebensvorteil besitzen, stellt von der ansonsten praktisch gleich ungünstigen Prognose jüngerer und älterer Patienten einen Ausnahmefall dar.

Aus den Daten zum Überleben der Gesamtgruppe sollten sich am ehesten unterschiedliche Behandlungsstrategien für jüngere und ältere Patienten ableiten, neben der Berücksichtigung der Komorbidität, die weder in unserer noch anderen bisherigen Studien eine angemessene Berücksichtigung fand. Ramalingam und Mitarbeiter stellten jedoch ganz offen fest, daß in die Entscheidung über die Aggressivität eines Behandlungskonzeptes eben nicht nur Stadium und Allgemeinzustand, sondern auch der „philosophische Standpunkt“ von Arzt und Patient eingeht [11]. Wir können aus eigener Erfahrung die Feststellung der Autoren nur bestätigen, daß jüngere Patienten eher aggressiver therapiert werden als ältere, weil sie in einem besseren Allgemeinzustand sind, stärker das Bedürfnis nach Ausschöpfung aller zur Verfügung stehenden Therapieoptionen äußern und weil der Arzt Widerstände dagegen aufbaut, beim jungen Patienten realistische therapeutische Grenzen zu akzeptieren.

Es erscheint also dringend geboten, prospektive Daten zu einer ganzen Reihe von Einzelaspekten des Bronchialkarzinoms beim jungen Erwachsenen zu erheben. Basierend auf den methodischen Erfahrungen, die durch eine Studie des Instituts für Epidemiologie am GSF-Forschungszentrum für Umwelt und Gesundheit zur Risikosituation solcher Patienten gewonnen wurden [7], bereitet daher die Deutsche Lungenstiftung e.V. in Zusammenarbeit mit einer Reihe von Lungenfachkliniken und -abteilungen der Bundesrepublik Deutschland sowie dem GSF-Forschungszentrum eine langfristig angelegte Studie vor $^{1}$. In ihr soll versucht werden, klinische Charakteristika, Familienanamnese, Schadstoffexposition und Prognose bei jungen Patienten im Vergleich zu einer Stichprobe älterer Erkrankter miteinander in Beziehung zu setzen. Hieraus ergibt sich die Erwartung, neben dem präventiven Aspekt eine geeignetere Grundlage für die notwendige Besonderheit im Umgang mit jungen Lungenkrebspatienten zu schaffen.

Die Arbeit wurde unterstützt durch den Verein zur Förderung der Pneumologie und Thoraxchirurgie in Berlin-Heckeshorn e.V.

\footnotetext{
1 Für dieses Projekt wurde die Unterstützung durch die Deutsche Krebshilfe beantragt.
} 


\section{Literatur}

${ }^{1}$ Antkowiak JG, Regal AM, Takita H. Bronchogenic carcinoma in patients under age 40. Ann Thorac Surg 1989; 47: 391 - 393

2 Bourke W, Milstein D, Giura R, Donghi M, Luisetti M, Rubin A-HE, Smith LJ. Lung cancer in young adults. Chest 1992; 102: $1723-1729$

${ }^{3}$ Capewell S, Wathen CG, Sankaran R, Sudlow MF. Lung cancer in young patients. Respir Med 1992; 86: 499 - 502

${ }^{4}$ Charloux A, Rossignol M, Purohit A, Small D, Wolkove N, Pauli G, Quoix E, Kreisman H. International differences in epidemiology of lung adenocarcinoma. Lung Cancer 1997; 16: 133 - 143

${ }^{5}$ Janssen-Heijnen MLG, Nab HW, Reek van J, Heijden van den LH, Schipper R, Coebergh JWW. Striking changes in smoking behaviour and lung cancer incidence by histological type in SouthEast Netherlands, 1960 - 1991. Eur J Cancer 1995; 31: 949 - 952

${ }^{6}$ Jubelirer SJ, Wilson RA. Lung cancer in patients younger than 40 years of age. Cancer 1991; 677: 1436 - 1438

${ }^{7}$ Kreuzer M, Kreienbock L, Gerken M, Heinrich J, Bruske-Hohlfeld I, Müller KM, Wichmann HE. Risk factors for lung cancer in young adults. Am J Epidemiol 1998; 147: 1028 - 1037

${ }^{8}$ Loddenkemper B, Schönfeld N. Epidemiologie, Früherkennung und Prävention. In: Loddenkemper, R (Hrsg). Das Bronchialkarzinom und andere bronchopulmonale Tumoren. Stuttgart: Kohlhammer; 1998

${ }^{9}$ McDuffie HH, Klaassen DJ, Dosman JA. Characteristics of patients with primary lung cancer diagnosed at age 50 years or younger. Chest 1989; 96: 1298 - 1301

${ }^{10}$ Neumann G. Das epidemiologische Bild des Bronchialkarzinoms. Pneumologie 1993; 47: 678 - 685

${ }^{11}$ Ramalingam S, Pawlish K, Gadgeel S, Demers R, Kalemkerian GP. Lung cancer in young patients: Analysis of a surveillance, epidemiology, and end results database. J Clin Oncol 1998; 16: $651-657$

12 Shimono T, Hayashi T, Kimura M, Yada I, Namikawa S, Yuasa H, Kusagawa M. Surgical treatment of primary lung cancer in patients less than 40 years of age. J Clin Oncol 1994; 12: 981 985

${ }^{13}$ Sugio K, Ishida T, Kaneko S, Yokohama H, Sugimachi K. Surgically resected lung cancer in young adults. Ann Thorac Surg 1992; 53: $127-131$

14 Travis WD, Travis LB, Devasa SS. Lung cancer. Cancer 1995; 75 : $191-202$

\section{Dr. Nicolas Schönfeld}

Pneumologische Abt. II Lungenklinik Heckeshorn Zum Heckeshorn 33

14109 Berlin

E-mail: loddheck@zedat.fu-berlin.de 